\title{
PHYSICAL THERAPY PROGRAM IN PATIENTS WITH TRANSCATHETER AORTIC VALVE IMPLANTATION
}

\author{
Antoaneta Dimitrova, Zhasmina Koleva, Ivan Maznev, Nikolay Izov, \\ Daniela Lubenova, Kristin Grigorova-Petrova, Milena Nikolova
}

\begin{abstract}
Summary
Aim: To evaluate the effect of cardiac rehabilitation on functional status in patients with transcatheter aortic valve implantation (TAVI).

Material and methods: Ten patients referred for physiotherapy before and after TAVI underwent in-hospital and after discharge assessments to evaluate functional status and degree of autonomy.

Results and discussion: All patients had improvement in six minutes walking performance and were more independent in activities in daily living compared with baseline after three months regular therapeutic exercise sessions.

Conclusion: Complex therapeutic approach shows benefits in activities of daily living and walking abilities and is suitable for all patients with TAVI.
\end{abstract}

Keywords: cardiac rehabilitation, transcatheter aortic valve implantation

\section{Introduction}

Transcatheter aortic valve implantation (TAVI) is a new procedure that delivers a replacement valve in a similar way that a cardiac stent is implanted. The valves are expanded in place over the existing valve and immediately begins functioning. TAVI is an alternative method for aortic valve replacement for old and polymorbide patients with contraindications to surgical aortic valve replacement (SAVR) and high surgical risk of opening the chest cavity. The choice of proceeding with SAVR versus TAVI is based on multiple factors, including the surgical risk, patient frailty, comorbid conditions, and patient preferences and values. Concomitant severe coronary artery disease may also affect the optimal intervention because severe multivessel coronary disease may best be served by SAVR and coronary artery bypass graft surgery (CABG). Longer-term follow-up and additional randomized controlled trials have demonstrated that TAVI is equivalent to SAVR for severe symptomatic aortic stenosis (AS) when surgical risk is high. Thus, in patients with severe symptomatic AS who are unable to undergo surgical AVR because of a prohibitive surgical risk and who have an expected survival of $>1$ year after intervention, TAVR is recommended to improve survival and reduce symptoms. This decision should be made only after discussion with the patient about the expected benefits and possible complications of TAVR. Patients with severe AS are considered to have a prohibitive surgical risk if they have a predicted risk with surgery of death or major morbidity (all causes) $>50 \%$ at 30 days; disease affecting $\geq 3$ major organ systems that is not likely to improve postoperatively; or anatomic factors that preclude or increase the risk of cardiac surgery, such as a heavily calcified (eg, porcelain) aorta, prior radiation, or an arterial bypass graft adherent to the chest wall. TAVI is not recommended in patients in whom existing comorbidities would preclude the expected benefit from correction of AS (Nishimura, et al., 2014; Nishimura, et al., 2017).

It's important to lead a heart-healthy lifestyle in order to prevent complications after TAVI, which insists personal lifestyle changes as weight reduction, healthy eating, and regular physical activity. Cardiac rehabilitation is a customized in-hospital and outpatient program of exercise and education, designed to improve the recover the patients from a heart attack, other forms of heart disease or surgery or implantation. Patients after TAVI, are candidates for referral to exercise-based individually tailored physiotherapy (PT). Despite this, until 2013 no data have been available about the safety and the efficacy of a comprehensive rehabilitative period in these subjects. physiotherapy is a helpful tool to maintain independency for daily life activities and participation in socio-cultural life. A lot of patients a month after TAVI have limited activities of daily living. The goals of cardiac rehabilitation include establishing an individualized plan to help regain 
strength, preventing from worsening, reducing the risk of future heart problems, and improving functional status and health-related quality of life (Cribier, et al., 2002; Fauchere, et al., 2014; Lawrie, 2012).

The current study aims to determine if patients after TAVI do benefit from an in-patient and three months home-based rehabilitation program despite their frailty.

\section{Material and methods}

The patients (mean age $71.6 \pm 5$; NYHA $3.3 \pm 0.5$ ) referred for physiotherapy before and after TAVI in City Clinic, Sofia, underwent in-hospital and after discharge 3 months home-based physiotherapy. Inclusion criteria were: all TAVI patients above the age of 65; male or female; signed informed consent. Exclusion criteria were: unstable clinical condition - according to treating physician; handicap before the procedure; recent stroke; decompensate diabetes mellitus type II; severe cognitive decline.

Outcome measures were six minutes walking test (6 MWT), five times sit to stand (FTSTS), Borg scale, inspiratory capacity (IC) and Cumulative Illness Rating Scale (CIRS) which evaluate polymorbidity in adults and elderly people related to conducting $\mathrm{PT}$ procedures. The scale is validated for rehabilitation in geriatrics and measures the clinical burden of multiple concomitant illnesses in patients (Parmalee, et al., 1995).

The assessments were performed four times: before TAVI procedure, on the day of hospital discharge, on the $1^{\text {st }}$ and $3^{\text {rd }}$ month to evaluate functional abilities and degree of autonomy.

Physiotherapy depends on the functional status of the patients according NYHA classification for chronic heart failure. The patients were class III and class IV of NYHA classification. The purpose of the PT is to maintain both the general condition of the patients, the ability to perform the daily activities and to prevent complications. In-hospital PT sessions were performed twice a day for 7-9 days. The exercises were performed in supine position with a raised upper part of the body, in sitting or standing position. The methodology of physiotherapy includes the following tasks:

1. To relieve cardiac activity by including extra-cardiac factors: mobilizing the muscle pump through rhythmic exercises for the distal parts of the limbs and chest and diaphragm breathing. The biofeedback breathing device for inspiratory training (Coach2 Incentive Spirometer) is applied to all patients with the instruction to make 5 repetitions with 1 to $3 \mathrm{~min}$ rests between them, at least 4-5 times a day, every day of the week during hospital stay.

2. Maintaining the coronary circulation - via isometric exercises in circular mode.

3. Maintaining the cardiovascular activity to perform daily activities - gradual verticalization, exercises for strengthening the lower limbs, slow walking in the room or hospital corridor, stair climbing, enhancing independence in terms of performing functional and self-service activities.

Physiotherapeutical methodology aims to achieve improvement of collateral circulation and trophic of myocardium by exercises against resistance for large muscle groups and to increase functional abilities of the cardiovascular system (by chest and diaphragm breathing, active exercises for large muscle groups, isometric muscle exercises).

After discharge the patients were given a booklet with instructions for home-based exercise program and breathing training with feedback inspiratory training device Coach2 Incentive Spirometer.

An indication for discontinue of the exercising is arrhythmia, tachycardia, precordial pain or chest discomfort, a change in the breathing pattern with inclusion of additional respiratory muscles, paradoxal breathing, etc.; severe fatigue or dyspnea; patient's desire to discontinue.

\section{Results}

All patients had progressive improvement in physical tolerance, decreased levels of fatigue and dyspnea increased respiratory function and were more independent in activities in daily living compared with baseline after three months' regular therapeutic exercise sessions (Table 1).

The patients increased the walking distance with $150 \mathrm{~m}$ on the $3^{\text {rd }}$ month after hospital discharge (from $185 \pm 77$ to $335 \pm 49 \mathrm{~m}$ ) and simultaneously decreased the levels of fatigue and dyspnoea from $6.3 \pm 2.5$ to $3 \pm 2$ points. 
Improvement in the functional lower limb muscle strength was observed because the time for performing the FTSTS test decreased with $7 \mathrm{sec}$ on the $3^{\text {rd }}$ month compared to the condition before TAVI in mean values.
Inspiratory capacity is important indicator for respiratory dysfunction. There is an improvement of $633 \mathrm{ml}$ on the $3^{\text {rd }}$ month compared to the baseline in mean values.

Table 1. Changes in functional status (mean value and standard deviation) in the patients before and after treatment

\begin{tabular}{|c|c|c|c|c|}
\hline Test & $\begin{array}{c}\mathrm{X}_{1}(\text { mean val- } \\
\text { ue } \pm \mathrm{SD})\end{array}$ & $\mathrm{X}_{2}($ mean value $\pm \mathrm{SD})$ & $\mathrm{X}_{3}($ mean value $\pm \mathrm{SD})$ & $\begin{array}{c}\mathrm{X}_{4}(\text { mean val- } \\
\text { ue } \pm \mathrm{SD})\end{array}$ \\
\hline $6 \mathrm{MWT}$ (m) & $280 \pm 98$ & $185 \pm 77$ & $270 \pm 98$ & $335 \pm 49$ \\
\hline $\begin{array}{l}\text { Borg Scale } \\
\text { (points) }\end{array}$ & $9.6 \pm 0.5$ & $6.3 \pm 2.5$ & $3.6 \pm 1.1$ & $3 \pm 2$ \\
\hline FTSTS (sec) & $27 \pm 4$ & $22 \pm 2$ & $21 \pm 4$ & $19.5 \pm 0.7$ \\
\hline IC $(\mathrm{ml})$ & $1300 \pm 526$ & $1516.6 \pm 625$ & $1866.6 \pm 709$ & $1933 \pm 802$ \\
\hline CIRS (points) & $15 \pm 5$ & $14 \pm 5$ & $14 \pm 5$ & $13 \pm 5$ \\
\hline
\end{tabular}

$X_{1}$ - before TAVI; $X_{2}$ - on hospital discharge; $X_{3}-1^{\text {st }}$ month; $X_{4}-3^{\text {rd }}$ month; SD - standard deviation; $6 M W T$ - Six minutes walking test; FTSTS-Five times sit to stand; IC - Inspiratory capacity; CIRS - Cumulative Illness Rating Scale

The changes in the results of Cumulative Illness Rating Scale (range $0-56$ points; 0 points - no problem affecting a body system; 56 points - extremely severe condition affecting all body systems) shows a slight tendency of improvement of overall physical status of 2 points.

\section{Discussion}

The main goals of physiotherapy in geriatric frail patients after TAVI treatment and many complications and comorbidities are to increase the physiological response to the body's oxygen needs, to reduce the symptoms, to increase the patient's functional capabilities, to educate the patient in the healthy lifestyle, to enhance prevention of secondary complication and to improve daily life activities for better reintegration of the patient. It has been shown that proper and regular PT in these patients can improve their physical tolerance, respiration and to reduce complaints. Physiotherapy usually starts with a hemodynamically stable patient's condition, aiming at responding to complaints. PT sessions include exercises for respiratory muscles, exercises for muscles of the limbs, frequent change of body position with low intensity.

Russo, N., et al. (2014) compared the safety and efficacy of a structured, exercise-based program in octogenarians after a traditional SAVR vs. TAVI, and concluded that a short-term, supervised, ex- ercise-based cardiac rehabilitation is feasible, safe and effective in octogenarian patients after TAVI as well as after traditional surgery. An early PT exercise program enhances independence, mobility and functional capacity and should be encouraged in all patients. Long-term effects and prognostic relevance should be evaluated in future studies.

Yue, Li. et al., (2015) compare the improvement in the quality of life in the long-term follow-up of 12 to 24 months after transcatheter aortic valve implantation therapy for old people with severe aortic stenosis using different questionnaires and conclude that there are significant improvements for this group in health-related quality of life in the follow-up. The authors assessed the hypothesis that patients after TAVI benefit from PT, compared to patients after SAVR. They concluded that patients after TAVI benefit from cardiac rehabilitation despite their older age and comorbidities. PT is a helpful tool to maintain independency for daily life activities and participation in socio-cultural life. The same conclusion was made by Fauchère, I., et al., (2012) patients in the TAVI group were older and sicker than SAVR patients. Despite these differences, both patient groups did benefit in the same way from a post-acute in-patient rehabilitation program as assessed by 6-Minute Walking Tests and FIM scores.

Zanettini, R., Gatto, G., Mori, I., et al. (2014), con- 
ducted a study with 60 patients after TAVI who underwent a long term rehabilitation in an outpatient center. The reported results underline the important role of early in-hospital and need to continues after discharge from hospital for increasing physical tolerance according 6 MWT. During in hospital rehabilitation the walking distance increased with $65 \mathrm{~m}$ from $210 \pm 87$ to $275 \pm 97 \mathrm{~m}$ in mean values. The greater distance compared to our patients may be due to the longer in-hospital stay of 3 weeks and their better condition - NYHA class $1.8 \pm 0.4$. Similar results of changes in physical tolerance according 6 MWT reported Fauchère, I., et al., (2012) in their study. In a large multicenter study called PARTNER trial with 699 enrolled patients after TAVI the authors concluded that if walking distance of 6 MWT not improve at 6-month follow-up results could predict long-term mortality in those unable to walk, in slow walkers and in fast walkers after two years. Walking ability is an indicator of overall physical fitness because it requires integration of circulatory, respiratory, nervous, and musculoskeletal systems. Walking speed is an established marker of frailty in the general population and in cohorts with cardiovascular disease and polymorbidity. Frailty is highly prevalent in the TAVI population. Their study does not suggest that TAVI is useless in those who are unable to walk (mortality rate $43.3 \%$ at 2 years). One of the limitations of the study is the lack of detailed information concerning the reasons for inability to perform the $6 \mathrm{MWT}$ and whether differences in the reasons for not performing the test (i.e., overall immobility vs severe shortness of breath at rest) have different prognostic implications. The patients who survive 2 years after TAVI improved the walking distance (Green, Cohen, Genereux, et al., 2013).

The FTSTS test is very useful in assessing elderly and frail patients for lower limb muscle strength, to quantifying functional change of transitional movements and risk of falls. Normative values for community dwelling adults from 70 to 79 years are $10.0+3.1 \mathrm{sec}$ (Bohannon, 2006). The results of our patients decreased from $27 \pm 4$ to $19.5 \pm 0.7 \mathrm{sec}$ in mean values and their functional abilities have the tendency to get closer to the normative values.

Inspiratory training involves the training of the specific muscles including diaphragmatic and intercostal muscles. Feedback inspiratory training devices are used for improving pulmonary function and providing feedback about respiratory training to the patients with different diseases. The inspiratory capacity data in-hospital patients with stroke showed improvement for 10 days, period with mean $300 \mathrm{ml}$ (Grigorova-Petrova, et al., 2014). The inspiratory capacity data in our study showed improvement with mean $216 \mathrm{ml}$ during the hospital stay. The greater inspiratory capacity mean values are a result of improved inspiration. The early application of an incentive breathing device for voluntary respiratory training is applicable in patients with TAVI in clinical setting and after discharge. The improvement of respiratory function is important for prevention of congestive pneumonia and to improve oxygen supply.

\section{Conclusion}

Complex physiotherapeutic approach benefits the functional status and is considered to be appropriate for patients with TAVI.

\section{References}

Bohannon R.W. Reference values for the five-repetition SIT-TO-STAND TEST: A descriptive META-ANALYSIS of data from elders. Perc.l and Motor Skills: 103; 215-222, 2006

Cribier, A., Eltchaninoff, H., Bash, A., Borenstein, N., Tron, C., Bauer, F., et al. (2002), Percutaneous transcatheter implantation of an aortic valve prosthesis for calcific aortic stenosis, Circulation, Vol. 106, pp. 3006-8.

Fauchere, I., Weber, D., Maier, W., et al. (2014), Rehabilitation after TAVI compared to surgical aortic valve replacement, International Journal of Cardiology, Vol. 173, № 3, pp. 564-566

Green, P., Cohen, D., Genereux, P., et al. (2013), Relation between six-minute walk test performance and outcomes after transcatheter aortic valve implantation (from the PARTNER trial), Am J Cardiol, Vol. 112, pp. 700-706

Grigorova-Petrova, K., Lubenova, D., Dimitrova, A., Baldaranov, D., Lozeva J. (2014), Feasibility of early physical therapy program in-hospital patients with acute ischemic stroke, Maced J Med Sci, Vol. 2, № 3, pp. 452-455 Lawrie, G. (2012), Role of Transcatheter Aortic Valve Implantation (TAVI) Versus Conventional Aortic Valve Replacement in the Treatment of Aortic Valve Disease, Methodist Debakey Cardiovasc J, Vol. 8 № 2, pp. 4-8

Nishimura, R., Otto, C., Bonow, R., et al. (2014), AHA/ ACC Guideline for the Management of Patients with Valvular Heart Disease, Circulation, Vol. 129, pp. 1-235 Nishimura, R., Otto, C., Bonow, R., et al. (2017), AHA/ ACC Focused Update of the 2014 AHA/ACC Guideline for the Management of Patients with Valvular Heart Disease, Circulation, Vol. 135, pp. e1159-e1195

Parmalee, P., Thuras, P., Katz, I, et al. (1995), Validation of the Cumulative Illness Rating Scale in a geriatric res- 
ident population. J Am Geriatr Soc, Vol. 43, pp. 130-137 Russo, N., Compostella, L., Tarantini, G., et al. (2014), Cardiac rehabilitation after transcatheter versus surgical prosthetic valve implantation for aortic stenosis in the elderly. European Journal of Preventive Cardiology, Vol. 21 № 11, pp. 1324-1331

Yue, Li. (2015), Improvement in Quality of Life in Old People with Aortic Stenosis after Transcatheter Aortic Valve Implantation, Current Signal Transduction Therapy, Vol. 9 № 3, pp. 164-171

Zanettini, R., Gatto, G., Mori, I., et al. (2014), Cardiac re- habilitation and mid-term follow-up after transcatheter aortic valve implantation, Journal of Geriatric Cardiology, Vol. 11, pp. 279-285

\section{Address for correspondence:}

Antoaneta Dimitrova

Department of Physiotherapy and Rehabilitation

National Sports Academy "Vassil Levski", Sofia, Bulgaria mobile: +359892299763

Email: tonialllex@yahoo.com 